EPSC Abstracts

Vol. 15, EPSC2021-626, 2021, updated on 11 Jan 2022

https://doi.org/10.5194/epsc2021-626

Europlanet Science Congress 2021

(C) Author(s) 2022. This work is distributed under

the Creative Commons Attribution 4.0 License.

\title{
The multi-chord stellar occultation by (19521) Chaos on 2020 November 20
}

Mónica Vara-Lubiano ${ }^{1}$, Nicolás Morales ${ }^{1}$, Flavia Rommel $^{2,3}$, José Luis Ortiz ${ }^{1}$, Bruno Sicardy ${ }^{4}$, Pablo Santos-Sanz ${ }^{1}$, Damya Souami ${ }^{4,5}$, Felipe Braga-Ribas ${ }^{2,3,6}$, Jure Skvarč ${ }^{7}$, Emmanuel Jehin ${ }^{8}$, Miguel Rodriguez-Alarcon ${ }^{9,10}$, Javier Licandro ${ }^{9,10}$, Miquel Serra ${ }^{9,10}$, Enric Pallé ${ }^{9,10}$, Hannu Parviainen ${ }^{9,10}$, Roberto Vieira-Martins ${ }^{2,3}$, Josselin Desmars ${ }^{11,12}$, René Duffard ${ }^{1}$, Julio Camargo ${ }^{2,3}$, Jean Lecacheux ${ }^{4}$, and the Chaos occultation team*

${ }^{1}$ Instituto de Astrofísica de Andalucía, IAA-CSIC, Glorieta de la Astronomía s/n, 18008 Granada, Spain. (mvara@iaa.es)

${ }^{2}$ Observatório Nacional/MCTIC, R. General José Cristino 77, Bairro Imperial de São Cristóvão, Rio de Janeiro (RJ), Brazil.

${ }^{3}$ Laboratório Interinstitucional de e-Astronomia - LIneA \& INCTdo e-Universo, Rua Gal. José Cristino 77, Bairro Imperial de São

Cristóvão, Rio de Janeiro (RJ), Brazil.

${ }^{4}$ LESIA, Observatoire de Paris, Université PSL, CNRS, Sorbonne Université, Univ. Paris Diderot, Sorbonne Paris Cité, 5 place

JulesJanssen, 92195 Meudon, France.

${ }^{5}$ naXys, University of Namur, Rempart de la Vierge, Namur, B-5000, Belgium

${ }^{6}$ Federal University of Technology - Paraná (UTFPR/DAFIS), Rua Sete de Setembro, 3165, Curitiba (PR), Brazil.

${ }^{7}$ Isaac Newton Group of Telescopes

${ }^{8}$ Space sciences, Technologies \& Astrophysics Research (STAR) Institute, University of Liège, Allée du 6 Août 19,4000 Liège, BELGIUM

${ }^{9}$ Instituto de Astrofísica de Canarias

${ }^{10}$ Universidad de La Laguna

${ }^{11}$ Institut Polytechnique des Sciences Avancées IPSA, 63 boulevard de Brandebourg, F-94200 Ivry-sur-Seine, France.

${ }^{12}$ Institut de Mécanique Céleste et de Calcul des Éphémérides, IMCCE, Observatoire de Paris, PSL Research University, CNRS, Sorbonne Universités, UPMC Univ Paris 06, Univ. Lille, 77, Av. Denfert-Rochereau, F-75014 Paris, France.

${ }^{*}$ A full list of authors appears at the end of the abstract

Physical properties of Trans-Neptunian Objects (TNOs) have been of increasing interest in the last two decades, as these objects are considered to be among the least altered through the Solar System evolution, and thus preserve valuable information about its origin [1]. The study of these objects through the ground-based method of stellar occultations has risen in the last years, as this technique allows the determination of physical properties with considerably good accuracies $[2,3,4]$.

Here we present the results of the multi-chord stellar occultation of the GAIA source $3444789965847631104\left(\mathrm{~m}_{\mathrm{v}} \approx 16.8\right)$ by the TNO (19521) Chaos on 2020 November 20, which was predicted within our systematic programme on stellar occultations by TNOs and outer solar system bodies [5]. The prediction was updated with astrometric observations carried out two days before the event with the 1.23-m telescope at Calar Alto observatory in Almería, Spain, and it was favorable to the South of Europe. The campaign that we organized involved 19 observing sites and resulted in three positive detections, one of them obtained from the 4.2-m WHT telescope at La Palma, 11 negative detections, and 5 sites that could not observe due to bad weather.

We derived the instantaneous limb of Chaos by fitting the extremities of the positive chords to an 
ellipse to determine accurate size, shape, and geometric albedo for this object. The preliminary results give a slightly smaller area-equivalent diameter than the one derived from Herschel thermal data [6], but photometric observations of this object are still under analysis to complement and improve the results.

\section{References}

[1] Morbidelli, A., Levison, H. F., \& Gomes, R. 2008, ed. M. A. Barucci, H. Boehnhardt, D. P. Cruikshank, A. Morbidelli, R. Dotson, 275

[2] Ortiz, J. L., Sicardy, B., Braga-Ribas, F., et al. 2012, Nature, 491, 566

[3] Braga-Ribas, F., Sicardy, B., Ortiz, J. L., et al. 2013, Ap], 773, 26

[4] Ortiz, J.L., Santos-Sanz, P., Sicardy, B., et al. 2017, Nature, 550, 7675, pp. 219-223

[5] Camargo, J. I. B., Vieira-Martins, R., Assafin, M., et al. 2014, A\&A, 561, A37

[6] Vilenius, E., Kiss, C., Mommert, M., Müller, T., et al. 2012, A\&A, 541, A94

\section{Acknowledgements}

We acknowledge financial support from the State Agency for Research of the Spanish MCIU through the "Center of Excellence Severo Ochoa" award to the Instituto de Astrofísica de Andalucía (SEV-2017-0709). Part of the research leading to these results has received funding from the European Research Council under the European Community's H2020 (2014-2020/ERC Grant Agreement no. 669416 "LUCKY STAR"). M.V-L. acknowledges funding from Spanish project AYA2017-89637-R (FEDER/MICINN). P.S-S. acknowledges financial support by the Spanish grant AYA-RTI2018-098657-J-I00 “'LEO-SBNAF" (MCIU/AEI/FEDER, UE). This work has made use of data from the European Space Agency (ESA) mission Gaia (https://www.cosmos.esa.int/gaia), processed by the Gaia Data Processing and Analysis Consortium (DPAC, https://www.cosmos.esa.int/web/gaia/dpac/consortium). We are grateful to the CAHA and OSN staffs. This research is partially based on observations collected at the Centro Astronómico Hispano Alemán (CAHA) at Calar Alto, operated jointly by Junta de Andalucía and Consejo Superior de Investigaciones Científicas (IAA-CSIC). This research was also partially based on observation carried out at the Observatorio de Sierra Nevada (OSN) operated by Instituto de Astrofísica de Andalucía (CSIC). Partially based on observations made with the Tx40 telescope at the Observatorio Astrofísico de Javalambre in Teruel, a Spanish Infraestructura Cientifico-Técnica Singular (ICTS) owned, managed and operated by the Centro de Estudios de Física del Cosmos de Aragón (CEFCA). Tx40 is funded with the Fondos de Inversiones de Teruel (FITE).

Chaos occultation team: Estela Fernández-Valenzuela[13], Álvaro Álvarez-Candal[1], Bruno Morgado[4,2,3], Gustavo Benedetti-Rossi[4,3], Joana Marques Oliveira[4], Altair Ramos GomesJúnior[14], Rodrigo Boufleur[3], Stefano Mottola[15], Stephan Hellmich[15], Herman Mikuz[16,17], Andras Pàl[18], Csaba Kiss[18], Anna Marciniak[19], Krzysztof Kaminski[19], Monika K. Kaminska[19], Yücel Kılıç[20,21], Metin Altan[22], M. N. Bagiran[23], Ramón Iglesias-Marzoa[24], Hugo Gonzalez[25], Alberto Castro-Tirado[1], Emilio Jesús Fernandez-Garcia[1], Kamil Hornoch[26], Petr Pravec[26], Hana Kučáková[26], Kosmas Gazeas[27] [13] Florida Space Institute, University of Central Florida, 12354 Research Parkway, Partnership 1, Orlando, FL, USA [14] UNESP - São Paulo State University, Grupo de Dinâmica Orbital e Planetologia, CEP 12516-410, Guaratinguetá, SP, Brazil. [15] German Aerospace Center (DLR), Institute of Planetary Research, 12489 Berlin, Germany [16] Crni Vrh Observatory, Slovenia [17] Faculty of Mathematics and Physics, University of Ljubljana, Slovenia [18] Konkoly Observatory of the Hungarian Academy of Sciences, Bu-dapest, Hungary [19] Astronomical Observatory Institute, Faculty of Physics, Adam Mickiewicz University in Poznan [20] TÜBİTAK National Observatory, Akdeniz University Campus, 07058 Antalya, Turkey [21] Akdeniz University, Department of Space Sciences and Technologies, Antalya, Turkey [22] Department of Physics, Science Faculty, Eski $\square$ ehir Technical University, 26470, Eski $\square$ ehir, Turkey [23] Türksat Observatory, Gölbaşı, Ankara, Turkey [24] Observatorio Astrofísico de Javalambre, 
Centro de Estudios de Física del Cosmos de Aragón [25] Observatorio de Forcarei [26] Ondrejov Observatory [27] Section of Astrophysics, Astronomy and Mechanics, Department of Physics, National and Kapodistrian University of Athens, GR-15784 Zografos, Athens, Greece 